\title{
Existence Results for a Class of $p$-Laplacian Fractional Differential Equations with Integral Boundary Conditions
}

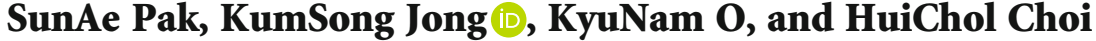 \\ Faculty of Mathematics, Kim Il Sung University, Pyongyang, Democratic People's Republic of Korea
}

Correspondence should be addressed to KumSong Jong; ksjong1016@163.com

Received 28 January 2020; Accepted 30 March 2020; Published 1 May 2020

Academic Editor: Patricia J. Y. Wong

Copyright (c) 2020 SunAe Pak et al. This is an open access article distributed under the Creative Commons Attribution License, which permits unrestricted use, distribution, and reproduction in any medium, provided the original work is properly cited.

\begin{abstract}
In this paper, we investigate the existence and uniqueness of solutions for a class of integral boundary value problems of nonlinear fractional differential equations with $p$-Laplacian operator. We obtain some existence and uniqueness results concerned with our problem by using Schaefer's fixed-point theorem and Banach contraction mapping principle. Finally, we present some examples to illustrate our main results.
\end{abstract}

\section{Introduction}

This paper deals with the existence and uniqueness of solutions for the following fractional integral boundary value problem with $p$-Laplacian operator:

$$
\left\{\begin{array}{l}
{ }^{c} D_{0+}^{\beta} \varphi_{p}\left({ }^{c} D_{0+}^{\alpha} x(t)\right)=f(t, x(t)), t \in(0,1), \\
x(0)=\int_{0}^{1} g(s) x(s) d s, x(1)=0, \\
\varphi_{p}\left({ }^{c} D_{0+}^{\alpha} x(0)\right)=\varphi_{p}\left({ }^{c} D_{0+}^{\alpha} x(1)\right)=\int_{0}^{1} h(s) \varphi_{p}\left({ }^{c} D_{0+}^{\alpha} x(s)\right) d s,
\end{array}\right.
$$

where $1<\alpha, \beta \leq 2,3<\alpha+\beta<4,{ }^{c} D_{0+}^{\alpha}$ and ${ }^{c} D_{0+}^{\beta}$ are the Caputo fractional derivatives, $f \in C([0,1] \times \mathbf{R}, \mathbf{R}), g, h \in$ $C([0,1], \mathbf{R})$, and the $p$-Laplacian operator is defined as $\varphi_{p}(s)=|s|^{p-2} s, p>1$.

In the past few decades, fractional differential equations have been widely applied to many fields in natural and social sciences, because they are important tools in mathematically describing many phenomena of science and engineering such as aerodynamics, control theory, signal and image processing, plasma dynamics, blood flow phenomena, and viscoelas- tic and non-Newtonian fluid mechanics (see [1-5] and their references). In 1983, Leibenson [2] proposed the following integer-order differential equation model with $p$-Laplacian operator to study the turbulent flow in a porous medium in his work:

$$
\left(\varphi_{p}\left(u^{\prime}(t)\right)\right)^{\prime}=f\left(t, u(t), u^{\prime}(t)\right), \quad t \in(0, \quad 1)
$$

where $\varphi_{p}$ is a $p$-Laplacian operator.

Based on Leibenson's results, many researchers have generalized his model into a variety of models with fractional order and obtained many valuable existence and uniqueness results for $p$-Laplacian fractional differential equations with two-point boundary conditions (see [6-14]), multipoint boundary conditions (see [15-18]), and nonlocal boundary conditions (see [19-23]). Since the $1^{\text {st }}$ derivatives of unknown functions existed in Leibenson's model, it became a special study to discuss the existence results for $p$-Laplacian fractional differential equations where the fractional orders were in the neighborhood of 1. So, Chai [7] used the fixed-point theorem on cones to investigate the existence and multiplicity of positive solutions for fractional differential equations with $p$-Laplacian operator: 


$$
\left\{\begin{array}{l}
D_{0+}^{\beta}\left(\varphi_{p}\left(D_{0+}^{\alpha} u(t)\right)\right)=-f(t, u(t)), \quad 0<t<1 \\
u(0)=0 \\
u(1)+\sigma D_{0+}^{\gamma} u(1)=0 \\
D_{0+}^{\alpha} u(0)=0
\end{array}\right.
$$

where $1<\alpha \leq 2,0<\beta, \gamma \leq 1$, and $D_{0+}^{\alpha}, D_{0+}^{\beta}$, and $D_{0+}^{\gamma}$ are the Riemann-Liouville fractional derivatives. Jong [15] established the existence and uniqueness of positive solutions for multipoint boundary value problems of nonlinear fractional differential equations with $p$-Laplacian operator by using the Banach contraction mapping principle

$$
\left\{\begin{array}{l}
D_{0+}^{\beta}\left(\varphi_{p}\left(D_{0+}^{\alpha} u(t)\right)\right)=f(t, \quad u(t)), \quad 0<t<1, \\
u(0)=0 \\
D_{0+}^{\gamma} u(1)=\sum_{i=1}^{m-2} \xi_{i} D_{0+}^{\gamma} u\left(\eta_{i}\right), \\
D_{0+}^{\alpha} u(0)=0, \\
\varphi_{p}\left(D_{0+}^{\alpha} u(1)\right)=\sum_{i=1}^{m-2} \zeta_{i} \varphi_{p}\left(D_{0+}^{\alpha} u\left(\eta_{i}\right)\right)
\end{array}\right.
$$

where $1<\alpha, \beta \leq 2,0<\gamma \leq 1$, and $f \in C([0,1] \times[0,+\infty)$, $[0,+\infty))$.

Integral boundary value problems for differential equations have arisen in the study of various fields such as underground water flow, blood flow problems, and thermoelasticity. So, the study on the existence of solutions for the $p$-Laplacian integral boundary value problems has attracted the attention of many researchers recently (see [20-23]). Zhang et al. [23] considered the existence of symmetric positive solutions of the problem for the following nonlinear fourth-order $p$-Laplacian differential equations with integral boundary conditions:

$$
\left\{\begin{array}{l}
\left(\varphi_{p}\left(x^{\prime \prime}(t)\right)\right)^{\prime \prime}=\omega(t) f(t, x(t)), \quad t \in(0,1), \\
x(0)=x(1)=\int_{0}^{1} g(s) x(s) d s, \\
\varphi_{p}\left(x^{\prime \prime}(0)\right)=\varphi_{p}\left(x^{\prime \prime}(1)\right)=\int_{0}^{1} h(s) \varphi_{p}\left(x^{\prime \prime}(s)\right) d s
\end{array}\right.
$$

where $\omega, g, h \in L[0,1]$ are the nonnegative, symmetric functions and $f \in C((0,1] \times[0,+\infty),[0,+\infty))$. Zhang and Cui [22] investigated the existence of positive solutions for nonlinear fourth-order singular $p$-Laplacian differential equations with the integral boundary conditions

$$
\left\{\begin{array}{l}
\left(\varphi_{p}\left(x^{\prime \prime}(t)\right)\right)^{\prime \prime}=f(t, x(t), x(t)), t \in(0,1), \\
x(0)=\int_{0}^{1} g(s) x(s) d s, x(1)=0, \\
\varphi_{p}\left(x^{\prime \prime}(0)\right)=\varphi_{p}\left(x^{\prime \prime}(1)\right)=\int_{0}^{1} h(s) \varphi_{p}\left(x^{\prime \prime}(s)\right) d s
\end{array}\right.
$$

where $f \in C((0,1) \times(0,+\infty) \times(0,+\infty),[0,+\infty)), f$ may be singular at $t=0,1, u=0$, and $g, h \in L[0,1]$ are nonnegative. The existence results on solutions of problem (2) are established by employing upper and lower solution methods together with maximal principle. Jiang [20] used the generalized continuous theorem to investigate the existence of solutions to the integral boundary value problem of $p$-Laplacian multiterm fractional differential equations at resonance

$$
\left\{\begin{array}{l}
D_{0+}^{\beta}\left(\varphi_{p}\left(D_{0+}^{\alpha} u(t)\right)\right)=-f\left(t, u(t), D_{0+}^{\alpha-1} u(t), D_{0+}^{\alpha} u(t)\right), 0<t<1, \\
u(0)=D_{0+}^{\alpha} u(0)=0, \\
u(1)=\int_{0}^{1} h(t) u(t) d t,
\end{array}\right.
$$

where $0<\beta \leq 1,1<\alpha \leq 2$, and $\int_{0}^{1} h(t) t^{\alpha-1} d t=1$.

Summarizing previous results, very few papers dealt with the existence of solutions for integral boundary value problems of $p$-Laplacian fractional differential equations, especially, Zhang and Cui [22] who established the existence of positive solutions for fourth-order singular $p$-Laplacian differential equations under $p \geq 2$ and Jiang [20] who considered the existence of solutions for nonlinear multiterm fractional differential equations with $0<\beta \leq 1$. Moreover, due to the nonlinearity of $p$-Laplacian operator $\varphi_{p}$, it is more difficult to study for the case $1<\beta \leq 2$ rather than for the case $0<\beta \leq 1$. Motivated by the above facts, this paper deals with the existence and uniqueness of solutions of problem (1) in which the fractional derivatives are Caputo fractional derivatives with $1<\alpha, \beta \leq 2$.The structure of this paper is organized as follows.

In Section 2, we recall some definitions and lemmas. In Section 3, we prove the existence and uniqueness of solutions for nonlinear integral boundary value problem with $p$-Laplacian operator by using Schaefer's fixed-point theorem and Banach contraction mapping principle. Finally, we give two examples to illustrate our main results in Section 4.

\section{Preliminaries}

The Riemann-Liouville fractional integral and the Caputo fractional derivative of order $\alpha>0$ of a function $f:(0, \infty)$ $\longrightarrow \mathbf{R}$ is given by 


$$
\begin{aligned}
\left(I_{0+}^{\alpha} f\right)(t) & :=\frac{1}{\Gamma(\alpha)} \int_{0}^{t}(t-s)^{\alpha-1} f(s) d s \\
\left({ }^{c} D_{0+}^{\alpha} f\right)(t) & :=\frac{1}{\Gamma(n-\alpha)} \int_{0}^{t}(t-s)^{n-\alpha-1} f^{(n)}(s) d s,
\end{aligned}
$$

where $n=[\alpha]+1$, provided that the right-hand side is pointwise defined on $(0, \infty)$ (see $[4,5])$.

Lemma 1 (see [1]). If $\alpha>0$ and $f \in C[a, b]$, then $\left({ }^{c} D_{a+}^{\alpha} I_{a+}^{\alpha} f\right)$ $(t)=f(t)$.

Lemma 2 (see [9]). Assume that ${ }^{c} D_{0+}^{\alpha} u \in C[0,1], \alpha>0$. Then, $I_{a+}^{\alpha}{ }^{c} D_{0+}^{\alpha} u(t)=u(t)+c_{0}+c_{1} t+\cdots c_{n-1} t^{n-1}, c_{i} \in \mathbf{R}, i=0,1, \cdots, n$ $-1, n-1<\alpha \leq n$.

For the sake of convenience, put $\sigma_{1}:=\int_{0}^{1}(1-s) g(s) d s$ and $\sigma_{2}:=\int_{0}^{1} h(s) d s$ and assume that $\sigma_{1} \neq 1$ and $\sigma_{2} \neq 1$.

Lemma 3. Let $\sigma \in C[0,1]$. Then, the fractional boundary value problem

$$
\left\{\begin{array}{l}
{ }^{c} D_{0+}^{\beta} z(t)=\sigma(t), \quad 0<t<1, \\
z(0)=z(1)=\int_{0}^{1} h(s) z(s) d s
\end{array}\right.
$$

has a unique solution which is given by

$$
z(t)=\frac{-1}{1-\sigma_{2}} \int_{0}^{1} \int_{0}^{1} h(s) G_{\beta}(s, \tau) \sigma(\tau) d \tau d s-\int_{0}^{1} G_{\beta}(t, s) \sigma(s) d s
$$

where

$$
G_{\beta}(t, s):=\frac{1}{\Gamma(\beta)}\left\{\begin{array}{l}
t(1-s)^{\beta-1}-(t-s)^{\beta-1}, 0 \leq s \leq t \leq 1 \\
t(1-s)^{\beta-1}, 0 \leq t \leq s \leq 1
\end{array}\right.
$$

Proof. In view of Lemma 2, we have that

$$
z(t)=c_{0}+c_{1} t+I_{0+}^{\beta} \sigma(t)
$$

By means of the property of the fractional integral of a continuous function, we obtain that $z(0)=c_{0}$.

Since $z(0)=z(1)=\int_{0}^{1} h(s) z(s) d s$, from (12), we obtain

$$
\begin{aligned}
& c_{0}=\int_{0}^{1} h(s) z(s) d s \\
& c_{1}=\int_{0}^{1} h(s) z(s) d s-\left.I_{0+}^{\beta} \sigma(t)\right|_{t=1}-\int_{0}^{1} h(s) z(s) d s=-\left.I_{0+}^{\beta} \sigma(t)\right|_{t=1} .
\end{aligned}
$$

Hence, (12) can be written as

$$
\begin{aligned}
z(t)= & \int_{0}^{1} h(s) z(s) d s+I_{0+}^{\beta} \sigma(t)-\left.I_{0+}^{\beta} \sigma(t)\right|_{t=1} \cdot t=\int_{0}^{1} h(s) z(s) d s \\
& +\frac{1}{\Gamma(\beta)}\left[\int_{0}^{t}(t-s)^{\beta-1} \sigma(s) d s-\int_{0}^{1} t(1-s)^{\beta-1} \sigma(s) d s\right]
\end{aligned}
$$

Thus, we can easily get

$$
z(t)=\int_{0}^{1} h(s) z(s) d s-\int_{0}^{1} G_{\beta}(t, s) \sigma(s) d s
$$

In the right side of (15), the term $\int_{0}^{1} h(s) z(s) d s$ can be rewritten as

$$
\begin{aligned}
\int_{0}^{1} h(s) z(s) d s & =\int_{0}^{1} h(s)\left(\int_{0}^{1} h(\tau) z(\tau) d \tau-\int_{0}^{1} G_{\beta}(s, \tau) \sigma(\tau) d \tau\right) d s \\
& =\int_{0}^{1} h(s) d s \int_{0}^{1} h(s) z(s) d s-\int_{0}^{1} \int_{0}^{1} h(s) G_{\beta}(s, \tau) \sigma(\tau) d \tau d s
\end{aligned}
$$

so we get

$$
\int_{0}^{1} h(s) z(s) d s=\frac{-1}{1-\sigma_{2}} \int_{0}^{1} \int_{0}^{1} h(s) G_{\beta}(s, \tau) \sigma(\tau) d \tau d s .
$$

Therefore, the unique solution of (9) is given by

$$
z(t)=\frac{-1}{1-\sigma_{2}} \int_{0}^{1} \int_{0}^{1} h(s) G_{\beta}(s, \tau) \sigma(\tau) d \tau d s-\int_{0}^{1} G_{\beta}(t, s) \sigma(s) d s
$$

Conversely, let $z \in C[0,1]$ be the function which is expressed by (10). Putting

$$
A(\beta):=\frac{-1}{1-\sigma_{2}} \int_{0}^{1} \int_{0}^{1} h(s) G_{\beta}(s, \tau) \sigma(\tau) d \tau d s,
$$

we get

$$
\begin{aligned}
z(t)= & A(\beta)-\int_{0}^{1} G_{\beta}(t, s) \sigma(s) d s=A(\beta)-\int_{0}^{t} G_{\beta}(t, s) \sigma(s) d s \\
& -\int_{t}^{1} G_{\beta}(t, s) \sigma(s) d s=A(\beta) \\
& -\frac{1}{\Gamma(\beta)}\left[\int_{0}^{t}\left(t(1-s)^{\beta-1}-(t-s)^{\beta-1}\right) \sigma(s) d s\right. \\
& \left.+\int_{t}^{1} t(1-s)^{\beta-1} \sigma(s) d s\right]=A(\beta) \\
& -\frac{1}{\Gamma(\beta)} \int_{0}^{1} t(1-s)^{\beta-1} \sigma(s) d s+\frac{1}{\Gamma(\beta)} \int_{0}^{t}(t-s)^{\beta-1} \sigma(s) d s .
\end{aligned}
$$


Then, we have that

$$
z(t)=A(\beta)-\left.t I_{0+}^{\beta} \sigma(t)\right|_{t=1}+I_{0+}^{\beta} \sigma(t)
$$

Since $\sigma \in C[0,1]$, applying ${ }^{c} D_{0+}^{\beta}$ to both sides of (21) and using Lemma 1 , we can obtain

$$
{ }^{c} D_{0+}^{\beta} z(t)={ }^{c} D_{0+}^{\beta} I_{0+}^{\beta} \sigma(t)=\sigma(t) .
$$

On the other hand, multiplying (10) by $h(t)$ and integrating on $[0,1]$, we have

$$
\begin{gathered}
\int_{0}^{1} z(t) h(t) d t=\frac{-1}{1-\sigma_{2}} \int_{0}^{1} h(t) d t \int_{0}^{1} \int_{0}^{1} h(s) G_{\beta}(s, \tau) \sigma(\tau) d \tau d s \\
-\int_{0}^{1} h(t) \int_{0}^{1} G_{\beta}(t, s) \sigma(s) d s d t=-\frac{\sigma_{2}}{1-\sigma_{2}} \int_{0}^{1} \int_{0}^{1} h(s) G_{\beta}(s, \tau) \sigma(\tau) \\
\cdot d \tau d s-\int_{0}^{1} \int_{0}^{1} h(t) G_{\beta}(t, s) \sigma(s) \\
\cdot d s d t=-\frac{1}{1-\sigma_{2}} \int_{0}^{1} \int_{0}^{1} h(s) G_{\beta}(s, \tau) \sigma(\tau) d \tau d s .
\end{gathered}
$$

Hence, (10) can be written as

$$
z(t)=-\int_{0}^{1} G_{\beta}(t, s) \sigma(s) d s+\int_{0}^{1} h(s) z(s) d s .
$$

Since $G_{\beta}(0, s)=G_{\beta}(1, s)=0$, we can also have that

$$
z(0)=z(1)=\int_{0}^{1} h(s) z(s) d s
$$

Therefore, we can know that $z(t)$ is a solution of problem (9) and (9) has a unique solution which is given by (10). The proof is completed.

Lemma 4. Let $f \in C([0,1] \times \mathbf{R}, \mathbf{R})$, then $p$-Laplacian integral boundary value problem (1) has a unique solution which is given by

$$
\begin{aligned}
x(t)= & \int_{0}^{1} G_{\alpha}(t, s) \varphi_{q}\left(\frac{1}{1-\sigma_{2}} \int_{0}^{1} \int_{0}^{1} h(\gamma) G_{\beta}(\gamma, \tau) f(\tau, x(\tau))\right. \\
& \left.\cdot d \tau d \gamma+\int_{0}^{1} G_{\beta}(s, \gamma) f(\gamma, x(\gamma)) d \gamma\right) \\
& \cdot d s+\frac{1-t}{1-\sigma_{1}} \int_{0}^{1} \int_{0}^{1} G_{\alpha}(\varsigma, s) g(\varsigma) \varphi_{q} \\
& \cdot\left(\frac{1}{1-\sigma_{2}} \cdot \int_{0}^{1} \int_{0}^{1} h(\gamma) G_{\beta}(\gamma, \tau) f(\tau, x(\tau)) d \tau d \gamma\right. \\
& \left.+\int_{0}^{1} G_{\beta}(s, \gamma) f(\gamma, x(\gamma)) d \gamma\right) d s d \varsigma
\end{aligned}
$$

where $q$ is the number that satisfies $1 / p+1 / q=1$.
Proof. The proof of this lemma is divided into two steps.

(Step 1) Let $r \in C[0,1]$ and consider the boundary value problem

$$
\left\{\begin{array}{l}
{ }^{c} D_{0+}^{\alpha} x(t)=r(t), \quad 0<t<1, \\
x(0)=\int_{0}^{1} g(s) x(s) d s, \quad x(1)=0 .
\end{array}\right.
$$

By using Lemma 2, we have that

$$
x(t)=d_{0}+d_{1} t+I_{0+}^{\alpha} r(t)
$$

From (28) and boundary condition of (27) we can obtain

$$
\begin{aligned}
& d_{0}=x(0)=\int_{0}^{1} g(s) x(s) d s, \\
& d_{1}=-d_{0}-\left.I_{0+}^{\alpha} r(t)\right|_{t=1}=-\int_{0}^{1} g(s) x(s) d s-\left.I_{0+}^{\alpha} r(t)\right|_{t=1} .
\end{aligned}
$$

Then, (28) can be written as

$$
\begin{aligned}
x(t)= & d_{0}+d_{1} t+I_{0+}^{\alpha} r(t)=\int_{0}^{1} g(s) x(s) \\
& \cdot d s+\left(-\int_{0}^{1} g(s) x(s) d s-\left.I_{0+}^{\alpha} r(t)\right|_{t=1}\right) t+I_{0+}^{\alpha} r(t) \\
& =I_{0+}^{\alpha} r(t)-\left.I_{0+}^{\alpha} r(t)\right|_{t=1} t+(1-t) \int_{0}^{1} g(s) x(s) \\
& \cdot d s=\frac{1}{\Gamma(\alpha)}\left[\int_{0}^{t}(t-s)^{\alpha-1} r(s) d s-\int_{0}^{1} t(1-s)^{\alpha-1} r(s) d s\right] \\
& +(1-t) \int_{0}^{1} g(s) x(s) d s=-\int_{0}^{1} G_{\alpha}(t, s) r(s) \\
& \cdot d s+(1-t) \int_{0}^{1} g(s) x(s) d s .
\end{aligned}
$$

In a similar way to the proof of Lemma 3, it can be easily seen that

$$
\int_{0}^{1} g(s) x(s) d s=\frac{-1}{1-\sigma_{1}} \int_{0}^{1} \int_{0}^{1} g(s) G_{\alpha}(s, \tau) r(\tau) d \tau d s .
$$

Hence, we have that the unique solution of (27) is given by $x(t)=-\int_{0}^{1} G_{\alpha}(t, s) r(s) d s-\frac{1-t}{1-\sigma_{1}} \int_{0}^{1} \int_{0}^{1} g(s) G_{\alpha}(s, \tau) r(\tau) d \tau d s$.

Conversely, let $x(t)$ be the function which is expressed by (32). Then, from the definition of $G_{\alpha}(t, s),(32)$ can be written as 
$x(t)=-\left.t I_{0+}^{\alpha} r(t)\right|_{t=1}+I_{0+}^{\alpha} r(t)-\frac{1-t}{1-\sigma_{1}} \int_{0}^{1} \int_{0}^{1} g(s) G_{\alpha}(s, \tau) r(\tau) d \tau d s$.

Since $r \in C[0,1]$ and $1<\alpha \leq 2$, applying ${ }^{c} D_{0+}^{\alpha}$ to both sides of (33), we can obtain

$$
{ }^{c} D_{0+}^{\alpha} x(t)={ }^{c} D_{0+}^{\alpha} I_{0+}^{\alpha} r(t)=r(t)
$$

On the other hand, multiplying (32) by $g(t)$ and integrating on $[0,1]$, we have that

$$
\begin{aligned}
\int_{0}^{1} g(t) x(t) d t= & -\int_{0}^{1} g(t) \int_{0}^{1} G_{\alpha}(t, s) r(s) \\
& \cdot d s d t-\frac{1}{1-\sigma_{1}} \int_{0}^{1}(1-t) g(t) \\
& \cdot d t \int_{0}^{1} \int_{0}^{1} g(s) G_{\alpha}(s, \tau) r(\tau) \\
& \cdot d \tau d s=-\int_{0}^{1} \int_{0}^{1} G_{\alpha}(t, s) g(t) r(s) \\
& \cdot d s d t-\frac{\sigma_{1}}{1-\sigma_{1}} \int_{0}^{1} \int_{0}^{1} g(s) G_{\alpha}(s, \tau) r(\tau) \\
& \cdot d \tau d s=-\left(1+\frac{\sigma_{1}}{1-\sigma_{1}}\right) \int_{0}^{1} \int_{0}^{1} G_{\alpha}(t, s) g(t) r(s) \\
& \cdot d s d t=\frac{-1}{1-\sigma_{1}} \int_{0}^{1} \int_{0}^{1} G_{\alpha}(t, s) g(t) r(s) d s d t .
\end{aligned}
$$

So, we can rewrite (32) as

$$
x(t)=-\int_{0}^{1} G_{\alpha}(t, s) r(s) d s+(1-t) \int_{0}^{1} g(t) x(t) d t
$$

Since $G_{\alpha}(0, s)=G_{\alpha}(1, s)=0$, we can get

$$
x(0)=\int_{0}^{1} g(t) x(t) d t, \quad x(1)=0 .
$$

Therefore, we can know that $x(t)$ is the solution of (27).

(Step 2) Now let $x(t)$ be the solution of (1). Putting $y(t)$ $:={ }^{c} D_{0+}^{\alpha} x(t)$, then we have that

$$
x(t)=-\int_{0}^{1} G_{\alpha}(t, s) y(s) d s-\frac{1-t}{1-\sigma_{1}} \int_{0}^{1} \int_{0}^{1} g(s) G_{\alpha}(s, \tau) y(\tau) d \tau d s .
$$

Also denoting $\varphi_{p}(y(t))$ by $z(t)$, then by Lemma 3 , we can see that that

$$
\begin{aligned}
z(t)= & \frac{-1}{1-\sigma_{2}} \int_{0}^{1} \int_{0}^{1} h(s) G_{\beta}(s, \tau) f(\tau, x(\tau)) \\
& \cdot d \tau d s-\int_{0}^{1} G_{\beta}(t, s) f(s, x(s)) d s .
\end{aligned}
$$

Since it is well-known that $\varphi_{p}^{-1}=\varphi_{q}$, combining (38) and (39) yields

$$
\begin{aligned}
x(t)= & \int_{0}^{1} G_{\alpha}(t, s) \varphi_{p}^{-1}\left(\frac{1}{1-\sigma_{2}} \int_{0}^{1} \int_{0}^{1} h(\gamma) G_{\beta}(\gamma, \tau) f(\tau, x(\tau))\right. \\
& \left.\cdot d \tau d \gamma+\int_{0}^{1} G_{\beta}(s, \gamma) f(\gamma, x(\gamma)) d \gamma\right) \\
& \cdot d s+\frac{1-t}{1-\sigma_{1}} \int_{0}^{1} \int_{0}^{1} G_{\alpha}(\varsigma, s) g(\varsigma) \varphi_{p}^{-1} \\
& \cdot\left(\frac{1}{1-\sigma_{2}} \cdot \int_{0}^{1} \int_{0}^{1} h(\gamma) G_{\beta}(\gamma, \tau) f(\tau, x(\tau)) d \tau d \gamma\right. \\
& \left.+\int_{0}^{1} G_{\beta}(s, \gamma) f(\gamma, x(\gamma)) d \gamma\right) d s d \varsigma .
\end{aligned}
$$

The proof is completed.

Remark 5. From the definition of $G_{\alpha}(t, s)$ and $G_{\beta}(t, s)$, it is easy to know that those functions are continuous in $[0,1] \times$ $[0,1]$.

Lemma 6 (see [24]). Schaefer's fixed-point theorem. Let $X$ be the Banach space and $T: X \longrightarrow X$ be completely continuous operator. If the set $E:=\{u \in X \mid u=\rho T u, 0<\rho<1\}$ is bounded, then $T$ has at least one fixed point in $X$.

The basic properties of the $p$-Laplacian operator which will be used in the following studies are listed below (see [10]).

(i) If $1<p \leq 2, x y>0$, and $|x|,|y| \geq m>0$, then

$$
\left|\varphi_{p}(x)-\varphi_{p}(y)\right| \leq(p-1) m^{p-2}|x-y|
$$

(ii) If $p>2$ and $|x|,|y| \leq M$, then

$$
\left|\varphi_{p}(x)-\varphi_{p}(y)\right| \leq(p-1) M^{p-2}|x-y|
$$

\section{Main Results}

In this section, we establish the existence and uniqueness of solutions of problem (1) by using Schaefer's fixed-point theorem and the Banach contraction mapping principle.

Let us consider the Banach space $X=C[0,1]$ endowed with the norm $\|u\|:=\max _{0 \leq t \leq 1}|u(t)|$. 
Define an operator $T: X \longrightarrow X$ by

$$
\begin{aligned}
T x(t):= & \int_{0}^{1} G_{\alpha}(t, s) \varphi_{q}\left(\frac{1}{1-\sigma_{2}} \int_{0}^{1} \int_{0}^{1} h(\gamma) G_{\beta}(\gamma, \tau) f(\tau, x(\tau))\right. \\
& \left.\cdot d \tau d \gamma+\int_{0}^{1} G_{\beta}(s, \gamma) f(\tau, x(\tau)) d \tau\right) \\
& \cdot d s+\frac{1-t}{1-\sigma_{1}} \int_{0}^{1} \int_{0}^{1} G_{\alpha}(\varsigma, s) g(\varsigma) \\
& \cdot \varphi_{q}\left(\frac{1}{1-\sigma_{2}} \cdot \int_{0}^{1} \int_{0}^{1} h(\gamma) G_{\beta}(\gamma, \tau) f(\tau, x(\tau))\right. \\
& \left.\cdot d \tau d \gamma+\int_{0}^{1} G_{\beta}(s, \gamma) f(\gamma, x(\gamma)) d \gamma\right) d s+\frac{1-t}{1-\sigma_{1}} .
\end{aligned}
$$

Then, Equation (26) is equivalent to the operator equation

$$
x=T x, x \in C[0,1] .
$$

From Lemma 4, the existence of solutions for the problem (1) refers to the existence of fixed points of Equation (44). Therefore, it is sufficient to prove the existence of fixed points of (44).

Lemma 7. The operator $T$ is completely continuous.

Proof. Since $f \in C([0,1] \times \mathbf{R}, \mathbf{R})$ and $\varphi_{q}$ is continuous, we can know that $T: X \longrightarrow X$ is continuous.

Let $\Omega \subset X$ be a bounded subset, then for any $u \in \Omega$, there exists $M_{0}>0$ such that $\|u\| \leq M_{0}$.

We will show that $T(\Omega)$ is relatively compact in $X$. Since $f$ is a continuous function, there exists $M_{f}>0$ such that $|f(t, u(t))| \leq M_{f}, t \in[0,1], u \in \Omega$. Then, we have

$$
\begin{aligned}
|T x(t)| \leq & \int_{0}^{1}\left|G_{\alpha}(t, s)\right| \cdot \mid \varphi_{q}\left(\frac{1}{1-\sigma_{2}} \int_{0}^{1} \int_{0}^{1} h(\gamma) G_{\beta}(\gamma, \tau) f(\tau, x(\tau))\right. \\
& \cdot d \tau d \gamma+\int_{0}^{1} G_{\beta}(s, \gamma) f(\gamma, x(\gamma)) d \gamma \mid \\
& \cdot d s+\frac{1-t}{1-\sigma_{1}} \int_{0}^{1} \int_{0}^{1}\left|G_{\alpha}(\varsigma, s) g(\varsigma)\right| \\
& \cdot \mid \varphi_{q}\left(\frac{1}{1-\sigma_{2}} \cdot \int_{0}^{1} \int_{0}^{1} h(\gamma) G_{\beta}(\gamma, \tau) f(\tau, x(\tau))\right. \\
& \left.\cdot d \tau d \gamma+\int_{0}^{1} G_{\beta}(s, \gamma) f(\gamma, x(\gamma)) d \gamma\right) \mid \\
& \cdot d s d \varsigma \leq \int_{0}^{1}\left|G_{\alpha}(t, s)\right| \\
& \cdot \mid \varphi_{q}\left(\frac{1}{1-\sigma_{2}} \int_{0}^{1} \int_{0}^{1} h(\gamma) G_{\beta}(\gamma, \tau) f(\tau, x(\tau))\right. \\
& \left.\cdot d \tau d \gamma+\int_{0}^{1} G_{\beta}(s, \gamma) f(\gamma, x(\gamma)) d \gamma\right) \mid \\
& \cdot d s+\frac{1-t}{1-\sigma_{1}} \int_{0}^{1} \int_{0}^{1}\left|G_{\alpha}(\varsigma, s) g(\varsigma)\right| \\
& \cdot \varphi_{q}\left(\frac{1}{1-\sigma_{2}} \cdot \int_{0}^{1} \int_{0}^{1} \mid h(\gamma) G_{\beta}(\gamma, \tau) f(\tau, x(\tau))\right. \\
& \left.\cdot\left|d \tau d \gamma+\int_{0}^{1}\right| G_{\beta}(s, \gamma) f(\gamma, x(\gamma)) \mid d \gamma\right) d s d \varsigma .
\end{aligned}
$$

And since $(t, s) \in[0,1] \times[0,1]$, evaluating the upper bound of $\left|G_{\beta}(t, s)\right|$ gives

$$
\left|G_{\beta}(t, s)\right| \leq \frac{1}{\Gamma(\beta)} .
$$

So, we obtain

$$
\begin{gathered}
\frac{1}{1-\sigma_{2}} \int_{0}^{1} \int_{0}^{1}\left|h(\gamma) G_{\beta}(\gamma, \tau) f(\tau, x(\tau))\right| \\
\cdot d \tau d \gamma+\int_{0}^{1}\left|G_{\beta}(s, \gamma) f(\gamma, x(\gamma))\right| \\
\cdot d \gamma \leq \frac{M_{f}}{\Gamma(\beta)}\left(1+\frac{\|h\|}{\left|1-\sigma_{2}\right|}\right)=: M_{1} .
\end{gathered}
$$

Then, we can get easily that

$$
\begin{aligned}
|T x(t)| \leq & \int_{0}^{1}\left|G_{\alpha}(t, s)\right| \cdot \varphi_{q}\left(M_{1}\right) \\
& \cdot d s+\frac{1-t}{1-\sigma_{1}} \int_{0}^{1} \int_{0}^{1}\left|G_{\alpha}(\varsigma, s) g(\varsigma)\right| \cdot \varphi_{q}\left(M_{1}\right) \\
& \cdot d s d \varsigma \leq \frac{\varphi_{q}\left(M_{1}\right)}{\Gamma(\alpha)}\left(1+\frac{\|g\|}{\left|1-\sigma_{1}\right|}\right) .
\end{aligned}
$$

In view of the definition of the $p$-Laplacian operator, we have that

$$
\|T x\| \leq \frac{M_{1}^{q-1}}{\Gamma(\alpha)}\left(1+\frac{\|g\|}{\left|1-\sigma_{1}\right|}\right) .
$$

This shows that $T(\Omega)$ is uniformly bounded in $X$. For any $x \in \Omega, 0 \leq t_{1}<t_{2} \leq 1$, we have that

$$
\begin{aligned}
\mid T x\left(t_{2}\right)- & T x\left(t_{1}\right)|=| \int_{0}^{1} G_{\alpha}\left(t_{2}, s\right) \\
& \cdot \varphi_{q}\left(\frac{1}{1-\sigma_{2}} \int_{0}^{1} \int_{0}^{1} h(\gamma) G_{\beta}(\gamma, \tau) f(\tau, x(\tau))\right. \\
& \left.\cdot d \tau d \gamma+\int_{0}^{1} G_{\beta}(s, \gamma) f(\gamma, x(\gamma)) d \gamma\right) \\
& \cdot d s+\frac{1-t_{2}}{1-\sigma_{1}} \int_{0}^{1} \int_{0}^{1} G_{\alpha}(\varsigma, s) g(\varsigma) \\
& \cdot \varphi_{q}\left(\frac{1}{1-\sigma_{2}} \cdot \int_{0}^{1} \int_{0}^{1} h(\gamma) G_{\beta}(\gamma, \tau) f(\tau, x(\tau))\right. \\
& \left.\cdot d \tau d \gamma+\int_{0}^{1} G_{\beta}(s, \gamma) f(\gamma, x(\gamma)) d \gamma\right) \\
& \cdot d s d \varsigma-\int_{0}^{1} G_{\alpha}\left(t_{1}, s\right) \varphi_{q}\left(\frac{1}{1-\sigma_{2}} \int_{0}^{1} \int_{0}^{1} h(\gamma) G_{\beta}(\gamma, \tau)\right. \\
& \left.\cdot f(\tau, x(\tau)) d \tau d \gamma+\int_{0}^{1} G_{\beta}(s, \gamma) f(\gamma, x(\gamma)) d \gamma\right)
\end{aligned}
$$




$$
\begin{aligned}
& \cdot d s-\frac{1-t_{1}}{1-\sigma_{1}} \int_{0}^{1} \int_{0}^{1} G_{\alpha}(\varsigma, s) g(\varsigma) \\
& \cdot \varphi_{q}\left(\frac{1}{1-\sigma_{2}} \cdot \int_{0}^{1} \int_{0}^{1} h(\gamma) G_{\beta}(\gamma, \tau) f(\tau, x(\tau))\right. \\
& \left.\cdot d \tau d \gamma+\int_{0}^{1} G_{\beta}(s, \gamma) f(\gamma, x(\gamma)) d \gamma\right) \\
& \cdot d s d \varsigma|=| \int_{0}^{1}\left(G_{\alpha}\left(t_{2}, s\right)-G_{\alpha}\left(t_{1}, s\right)\right) \\
& \cdot \varphi_{q}\left(\frac{1}{1-\sigma_{2}} \int_{0}^{1} \int_{0}^{1} h(\gamma) G_{\beta}(\gamma, \tau) f(\tau, x(\tau))\right. \\
& \left.\cdot d \tau d \gamma+\int_{0}^{1} G_{\beta}(s, \gamma) f(\gamma, x(\gamma)) d \gamma\right) \\
& \cdot d s+\frac{t_{1}-t_{2}}{1-\sigma_{1}} \int_{0}^{1} \int_{0}^{1} G_{\alpha}(\varsigma, s) g(\varsigma) \\
& \cdot \varphi_{q}\left(\frac{1}{1-\sigma_{2}} \cdot \int_{0}^{1} \int_{0}^{1} h(\gamma) G_{\beta}(\gamma, \tau) f(\tau, x(\tau))\right. \\
& \left.\cdot d \tau d \gamma+\int_{0}^{1} G_{\beta}(s, \gamma) f(\gamma, x(\gamma)) d \gamma\right) \\
& \cdot d s d \varsigma \leq M_{1}^{q-1} \cdot \int_{0}^{1}\left|G_{\alpha}\left(t_{2}, s\right)-G_{\alpha}\left(t_{1}, s\right)\right| \\
& \cdot d s+M_{1}^{q-1} \cdot \frac{t_{2}-t_{1}}{\left|1-\sigma_{1}\right|} \frac{\|g\|}{\Gamma(\alpha)} \cdot
\end{aligned}
$$

And since

$G_{\alpha}\left(t_{2}, s\right)-G_{\alpha}\left(t_{1}, s\right)=\frac{1}{\Gamma(\alpha)}\left\{\begin{array}{l}t_{2}(1-s)^{\alpha-1}-\left(t_{2}-s\right)^{\alpha-1}-t_{1}(1-s)^{\alpha-1}+\left(t_{1}-s\right)^{\alpha-1}, \quad s \leq t_{1}, \\ t_{2}(1-s)^{\alpha-1}-\left(t_{2}-s\right)^{\alpha-1}-t_{1}(1-s)^{\alpha-1}, \quad t_{1} \leq s \leq t_{2}, \\ t_{2}(1-s)^{\alpha-1}-t_{1}(1-s)^{\alpha-1}, \quad s \geq t_{2},\end{array}\right.$

the integral term $\int_{0}^{1}\left|G_{\alpha}\left(t_{2}, s\right)-G_{\alpha}\left(t_{1}, s\right)\right| d s$ can be divided into the following three parts:

$$
\begin{aligned}
& \int_{0}^{t_{1}}\left|G_{\alpha}\left(t_{2}, s\right)-G_{\alpha}\left(t_{1}, s\right)\right| \\
& \cdot d s=\frac{1}{\Gamma(\alpha)} \int_{0}^{t_{1}} \mid t_{2}(1-s)^{\alpha-1}-\left(t_{2}-s\right)^{\alpha-1} \\
& -\left(t_{1}(1-s)^{\alpha-1}-\left(t_{1}-s\right)^{\alpha-1}\right) \mid \\
& \cdot d s \leq \frac{1}{\Gamma(\alpha)} \int_{0}^{t_{1}}\left(\left(t_{2}-t_{1}\right)(1-s)^{\alpha-1}+\left(\left(t_{2}-s\right)^{\alpha-1}\right.\right. \\
& \left.-\left(t_{1}-s\right)^{\alpha-1}\right) d s \leq \frac{1}{\Gamma(\alpha)}\left(t_{2}-t_{1}\right) \int_{0}^{t_{1}}(1-s)^{\alpha-1} \\
& \cdot d s+\frac{1}{\Gamma(\alpha)} \int_{0}^{t_{1}}\left(\left(t_{2}-s\right)^{\alpha-1}-\left(t_{1}-s\right)^{\alpha-1}\right) \\
& \cdot d s \leq \frac{t_{2}-t_{1}}{\Gamma(\alpha+1)}+\frac{\left(t_{2}^{\alpha}-t_{1}^{\alpha}\right)-\left(t_{2}-t_{1}\right)^{\alpha}}{\Gamma(\alpha+1)} .
\end{aligned}
$$

In a similar way to this, we can obtain

$$
\begin{aligned}
& \int_{t_{1}}^{t_{2}}\left|G_{\alpha}\left(t_{2}, s\right)-G_{\alpha}\left(t_{1}, s\right)\right| d s \leq \frac{t_{2}-t_{1}}{\Gamma(\alpha+1)}+\frac{\left(t_{2}-t_{1}\right)^{\alpha}}{\Gamma(\alpha+1)}, \\
& \int_{t_{2}}^{1}\left|G_{\alpha}\left(t_{2}, s\right)-G_{\alpha}\left(t_{1}, s\right)\right| d s \leq \frac{t_{2}-t_{1}}{\Gamma(\alpha+1)} .
\end{aligned}
$$

These inequalities yield

$$
\begin{aligned}
& \int_{0}^{1}\left|G_{\alpha}\left(t_{2}, s\right)-G_{\alpha}\left(t_{1}, s\right)\right| \\
& \cdot d s=\int_{0}^{t_{1}}\left|G_{\alpha}\left(t_{2}, s\right)-G_{\alpha}\left(t_{1}, s\right)\right| \\
& \cdot d s+\int_{t_{1}}^{t_{2}}\left|G_{\alpha}\left(t_{2}, s\right)-G_{\alpha}\left(t_{1}, s\right)\right| \\
& \cdot d s+\int_{t_{2}}^{1}\left|G_{\alpha}\left(t_{2}, s\right)-G_{\alpha}\left(t_{1}, s\right)\right| \\
& \cdot d s \leq \frac{3\left(t_{2}-t_{1}\right)+\left(t_{2}^{\alpha}-t_{1}^{\alpha}\right)}{\Gamma(\alpha+1)} .
\end{aligned}
$$

Therefore, we get

$$
\begin{aligned}
\mid T x\left(t_{2}\right) & -T x\left(t_{1}\right) \mid \leq M_{1}^{q-1} \cdot \frac{3\left(t_{2}-t_{1}\right)+\left(t_{2}^{\alpha}-t_{1}^{\alpha}\right)}{\Gamma(\alpha+1)} \\
& +M_{1}^{q-1} \cdot \frac{t_{2}-t_{1}}{\left|1-\sigma_{1}\right|} \frac{\|g\|}{\Gamma(\alpha)}=\frac{M_{1}^{q-1}}{\Gamma(\alpha)}\left(\frac{3}{\alpha}+\frac{\|g\|}{\left|1-\sigma_{1}\right|}\right) \\
& \cdot\left(t_{2}-t_{1}\right)+\frac{M_{1}^{q-1}}{\Gamma(\alpha+1)} \cdot\left(t_{2}^{\alpha}-t_{1}^{\alpha}\right) .
\end{aligned}
$$

This shows that $T(\Omega)$ is equicontinuous in $X$. By using the Arzela-Ascoli theorem, we can see that $T(\Omega)$ is relatively compact in $X$. As a consequence of the above discussion, the operator $T: X \longrightarrow X$ is completely continuous. The proof is completed.

Denote as follows:

$$
A:=\left[\frac{1}{\Gamma(\alpha)}\left(1+\frac{\|g\|}{\left|1-\sigma_{1}\right|}\right)\right]^{p-1} \frac{1}{\Gamma(\beta)}\left(1+\frac{\|h\|}{\left|1-\sigma_{2}\right|}\right) .
$$

In this article, the following hypotheses will be used. (H1). There exist nonnegative functions $a, b \in C[0,1]$ such that

(i) $|f(t, x)| \leq a(t)+b(t)|x|^{p-1}, t \in[0,1], x \in \mathbf{R}$

(ii) $A\|b\|<1$

Theorem 8. Assume that the hypothesis (H1) holds, then problem (1) has at least one solution. 
Proof. Consider the following set:

$$
E:=\{x \in X \mid x=\rho T x, 0<\rho<1\} .
$$

For any $x \in E$, it can be easily seen that

$$
\|x\|=\rho\|T x\|<\|T x\| .
$$

On the other hand, from the condition (i) of the hypothesis (H1) and (49), we have that

$$
\begin{aligned}
\|T x\| \leq & \frac{1}{\Gamma(\alpha)}\left(1+\frac{\|g\|}{\left|1-\sigma_{1}\right|}\right) \\
& \cdot\left(\frac{1}{\Gamma(\beta)}\left(1+\frac{\|h\|}{\left|1-\sigma_{2}\right|}\right)\left(\|a\|+\|b\|\|x\|^{p-1}\right)\right)^{q-1} .
\end{aligned}
$$

From (58), we get

$$
\begin{aligned}
\|x\|< & \frac{1}{\Gamma(\alpha)}\left(1+\frac{\|g\|}{\left|1-\sigma_{1}\right|}\right) \\
& \cdot\left(\frac{1}{\Gamma(\beta)}\left(1+\frac{\|h\|}{\left|1-\sigma_{2}\right|}\right)\left(\|a\|+\|b\|\|x\|^{p-1}\right)\right)^{q-1} .
\end{aligned}
$$

Since $1 / p+1 / q=1$ implies $(p-1)(q-1)=1$, we obtain

$$
\|x\|^{p-1}<A\left(\|a\|+\|b\|\|x\|^{p-1}\right)
$$

Therefore, we have that

$$
\|x\|^{p-1}(1-A\|b\|)<A\|a\|
$$

and by using the condition (ii) of the hypothesis (H1), we can see that

$$
\|x\|<\left(\frac{A\|a\|}{1-A\|b\|}\right)^{q-1} .
$$

So, we can know that $E$ is bounded. In view of Schaefer's fixed-point theorem (Lemma 6), the operator $T: X \longrightarrow X$ has at least one fixed point which is the solution of the problem (1). The proof is completed.

Here, put $r:=(A\|a\| / 1-A\|b\|)^{q-1}$ and list more hypotheses to obtain the uniqueness results for our problem.

(H2). There exists a real number $L>0$ such that

$$
|f(t, x)-f(t, y)|<L|x-y|, t \in[0,1], x, y \in[-r, r] .
$$

For the readers' convenience, denote as follows:

$$
\begin{aligned}
M_{0} & :=\left(1+\frac{\|h\|}{\left|1-\sigma_{2}\right|}\right)\left(\|a\|+\|b\| r^{p-1}\right), \\
\theta & :=\frac{L(q-1) M_{0}^{q-2}}{\Gamma(\alpha) \Gamma(\beta)}\left(1+\frac{\|g\|}{\left|1-\sigma_{1}\right|}\right)\left(1+\frac{\|h\|}{\left|1-\sigma_{2}\right|}\right) .
\end{aligned}
$$

Theorem 9. Let $1<p<2$. Assume that the hypotheses (H1) and (H2) are satisfied and $0<\theta<1$, then problem (1) has a unique solution.

Proof. Put $B=\{x \in X \mid\|x\| \leq r\}$.

Firstly, we will show that $T(B) \subset B$. In fact, from the hypothesis (H1) and (49), for any $x \in B$, we have that

$$
\begin{aligned}
\|T x\| \leq & \frac{1}{\Gamma(\alpha)}\left(1+\frac{\|g\|}{\left|1-\sigma_{1}\right|}\right)\left(\frac{1}{\Gamma(\beta)}\left(1+\frac{\|h\|}{\left|1-\sigma_{2}\right|}\right)\right. \\
& \left.\cdot\left(\|a\|+\|b\|\|x\|^{p-1}\right)\right)^{q-1} \\
= & {\left[\left(\frac{1}{\Gamma(\alpha)}\left(1+\frac{\|g\|}{\left|1-\sigma_{1}\right|}\right)\right)^{p-1} \frac{1}{\Gamma(\beta)}\right.} \\
& \left.\cdot\left(1+\frac{\|h\|}{\left|1-\sigma_{2}\right|}\right)\right]^{q-1}\left(\|a\|+\|b\|\|x\|^{p-1}\right)^{q-1} \\
\leq & A^{q-1}\left(\|a\|+\|b\| r^{p-1}\right)^{q-1} \\
= & A^{q-1}\left(\|a\|+\frac{A\|a\|\|b\|}{1-A\|b\|}\right)^{q-1} \\
= & \left(\frac{A\|a\|}{1-A\|b\|}\right)^{q-1}=r .
\end{aligned}
$$

Next, we will prove the uniqueness of solutions for problem (1)

For any $x \in B$ and any $t \in[0,1]$, by the hypothesis (H1), we get

$$
\begin{aligned}
& \frac{1}{\left|1-\sigma_{2}\right|} \int_{0}^{1} \int_{0}^{1} h(\gamma) G_{\beta}(\gamma, \tau) f(\tau, x(\tau)) \\
& \cdot d \tau d \gamma+\int_{0}^{1} G_{\beta}(s, \gamma) f(\gamma, x(\gamma)) \\
& \cdot d \gamma \leq \frac{\|h\|}{\left|1-\sigma_{2}\right|} \int_{0}^{1} \int_{0}^{1} G_{\beta}(\gamma, \tau) f(\tau, x(\tau)) \\
& \cdot d \tau d \gamma+\int_{0}^{1} G_{\beta}(s, \gamma) f(\gamma, x(\gamma)) \\
& \cdot d \gamma \leq \frac{\|h\|}{\left|1-\sigma_{2}\right|} \int_{0}^{1} \int_{0}^{1} G_{\beta}(\gamma, \tau)\left(a(\tau)+b(\tau) r^{p-1}\right) \\
& \cdot d \tau d \gamma+\int_{0}^{1} G_{\beta}(s, \gamma)\left(a(\gamma)+b(\gamma) r^{p-1}\right) \\
& \cdot d \gamma \leq\left(\frac{\|h\|}{\left|1-\sigma_{2}\right|}+1\right)\left(\|a\|+\|b\| r^{p-1}\right)=M_{0} .
\end{aligned}
$$

Since $1<p<2$, we can see $q>2$. Hence, from (H2) and one basic property of $p$-Laplacian operator (42), for any $x, y$ $\in B$ and any $t \in[0,1]$, we have that 


$$
\begin{aligned}
& |T x(t)-T y(t)|=\mid \int_{0}^{1} G_{\alpha}(t, s) \\
& \cdot \varphi_{q}\left(\frac{1}{1-\sigma_{2}} \int_{0}^{1} \int_{0}^{1} h(\gamma) G_{\beta}(\gamma, \tau) f(\tau, x(\tau)) d \tau d \gamma\right. \\
& \left.+\int_{0}^{1} G_{\beta}(s, \gamma) f(\gamma, x(\gamma)) d \gamma\right) d s+\frac{1-t}{1-\sigma_{1}} \int_{0}^{1} \int_{0}^{1} G_{\alpha}(\varsigma, s) g(\varsigma) \\
& \cdot \varphi_{q}\left(\frac{1}{1-\sigma_{2}} \cdot \int_{0}^{1} \int_{0}^{1} h(\gamma) G_{\beta}(\gamma, \tau) f(\tau, x(\tau))\right. \\
& \left.\cdot d \tau d \gamma+\int_{0}^{1} G_{\beta}(s, \gamma) f(\gamma, x(\gamma)) d \gamma\right) \\
& \cdot d s d \varsigma-\int_{0}^{1} G_{\alpha}(t, s) \varphi_{q}\left(\frac{1}{1-\sigma_{2}} \int_{0}^{1} \int_{0}^{1} h(\gamma) G_{\beta}(\gamma, \tau) f(\tau, y(\tau))\right. \\
& \left.\cdot d \tau d \gamma+\int_{0}^{1} G_{\beta}(s, \gamma) f(\gamma, y(\gamma)) d \gamma\right) \\
& \cdot d s-\frac{1-t}{1-\sigma_{1}} \int_{0}^{1} \int_{0}^{1} G_{\alpha}(\varsigma, s) g(\varsigma) \varphi_{q}\left(\frac{1}{1-\sigma_{2}} \cdot \int_{0}^{1} \int_{0}^{1} h(\gamma)\right. \\
& \text { - } \left.G_{\beta}(\gamma, \tau) f(\tau, y(\tau)) d \tau d \gamma+\int_{0}^{1} G_{\beta}(s, \gamma) f(\gamma, y(\gamma)) d \gamma\right) \\
& \cdot d s d \varsigma|\leq| \int_{0}^{1} G_{\alpha}(t, s)\left(\varphi _ { q } \left(\frac{1}{1-\sigma_{2}} \int_{0}^{1} \int_{0}^{1} h(\gamma) G_{\beta}(\gamma, \tau)\right.\right. \\
& \left.\cdot f(\tau, x(\tau)) d \tau d \gamma+\int_{0}^{1} G_{\beta}(s, \gamma) f(\gamma, x(\gamma)) d \gamma\right) \\
& \cdot d s-\varphi_{q}\left(\frac{1}{1-\sigma_{2}} \int_{0}^{1} \int_{0}^{1} h(\gamma) G_{\beta}(\gamma, \tau) f(\tau, y(\tau))\right. \\
& \left.\left.\cdot d \tau d \gamma+\int_{0}^{1} G_{\beta}(s, \gamma) f(\gamma, y(\gamma)) d \gamma\right)\right) \\
& \cdot d s+\frac{1-t}{1-\sigma_{1}} \int_{0}^{1} \int_{0}^{1} G_{\alpha}(\varsigma, s) g(\varsigma) \\
& \cdot\left(\varphi _ { q } \left(\frac{1}{1-\sigma_{2}} \cdot \int_{0}^{1} \int_{0}^{1} h(\gamma) G_{\beta}(\gamma, \tau) f(\tau, x(\tau))\right.\right. \\
& \left.\cdot d \tau d \gamma+\int_{0}^{1} G_{\beta}(s, \gamma) f(\gamma, x(\gamma)) d \gamma\right) \\
& \cdot-\varphi_{q}\left(\frac{1}{1-\sigma_{2}} \cdot \int_{0}^{1} \int_{0}^{1} h(\gamma) G_{\beta}(\gamma, \tau) f(\tau, y(\tau))\right. \\
& \left.\left.\cdot d \tau d \gamma+\int_{0}^{1} G_{\beta}(s, \gamma) f(\gamma, y(\gamma)) d \gamma\right)\right) \\
& \cdot d s d \varsigma\left|\leq \frac{(q-1) M_{0}^{q-2}}{\left|1-\sigma_{2}\right|} \int_{0}^{1}\right| G_{\alpha}(t, s)\left(\int_{0}^{1} \int_{0}^{1} h(\gamma) G_{\beta}(\gamma, \tau)\right. \\
& \cdot(f(\tau, x(\tau))-f(\tau, y(\tau))) d \tau d \gamma) \\
& \cdot d s+(q-1) M_{0}^{q-2} \int_{0}^{1} \mid G_{\alpha}(t, s) \int_{0}^{1} G_{\beta}(s, \gamma) \\
& \cdot(f(\gamma, x(\gamma))-f(\gamma, y(\gamma))) d \gamma \\
& \cdot d s+\frac{(1-t)(q-1) M_{0}^{q-2}}{\left|1-\sigma_{1}\right| \cdot\left|1-\sigma_{2}\right|} \cdot \int_{0}^{1} \int_{0}^{1} \mid G_{\alpha}(\varsigma, s) g(\varsigma)\left(\int_{0}^{1} \int_{0}^{1} h(\gamma)\right. \\
& \left.\cdot G_{\beta}(\gamma, \tau)(f(\tau, x(\tau))-f(\tau, y(\tau))) d \tau d \gamma\right) \\
& \cdot d s d \varsigma+\frac{(1-t)(q-1) M_{0}^{q-2}}{\left|1-\sigma_{1}\right|} \int_{0}^{1} \int_{0}^{1} \mid G_{\alpha}(\varsigma, s) g(\varsigma) \\
& \cdot\left(\int_{0}^{1} G_{\beta}(s, \gamma)(f(\gamma, x(\gamma))-f(\gamma, y(\gamma))) d \gamma\right) \mid
\end{aligned}
$$

$$
\begin{aligned}
& \cdot d s d \varsigma \leq \frac{L(q-1) M_{0}^{q-2}}{\left|1-\sigma_{2}\right|} \int_{0}^{1} G_{\alpha}(t, s) \\
& \cdot\left(\int_{0}^{1} \int_{0}^{1} h(\gamma) G_{\beta}(\gamma, \tau)|x(\tau)-y(\tau)| d \tau d \gamma\right) \\
& \cdot d s+L(q-1) M_{0}^{q-2} \int_{0}^{1} G_{\alpha}(t, s) \int_{0}^{1} G_{\beta}(s, \gamma)|x(\gamma)-y(\gamma)| \\
& \cdot d \gamma d s+\frac{L(1-t)(q-1) M_{0}^{q-2}}{\left|1-\sigma_{1}\right| \cdot\left|1-\sigma_{2}\right|} \int_{0}^{1} \int_{0}^{1} G_{\alpha}(\varsigma, s) g(\varsigma) \int_{0}^{1} \int_{0}^{1} h(\gamma) \\
& \cdot G_{\beta}(\gamma, \tau)|x(\tau)-y(\tau)| d \tau d \gamma d s d \varsigma+\frac{L(1-t)(q-1) M_{0}^{q-2}}{\left|1-\sigma_{1}\right|} \\
& \cdot \int_{0}^{1} \int_{0}^{1} G_{\alpha}(\varsigma, s) g(\varsigma) \int_{0}^{1} G_{\beta}(s, \gamma)|x(\gamma)-y(\gamma)| \\
& \cdot d \gamma d s d \varsigma \leq \frac{L(q-1) M_{0}^{q-2}}{\Gamma(\alpha) \Gamma(\beta)} \\
& \cdot\left(\frac{\|h\|}{\left|1-\sigma_{2}\right|}+1+\frac{\|g\|}{\left|1-\sigma_{1}\right|\left|1-\sigma_{2}\right|}+\frac{\|h\|}{\left|1-\sigma_{1}\right|}\right) \\
& \cdot\|x-y\|=\theta\|x-y\| \cdot
\end{aligned}
$$

This means that

$$
\|T x-T y\| \leq \theta\|x-y\| .
$$

Since $0<\theta<1$, we can see that $T: B \rightarrow B$ is a contraction mapping. By means of the Banach contraction mapping principle, we can prove that $T$ has a unique fixed point in $B$. That is, problem (1) has a unique solution. The proof is completed.

\section{Examples}

The following examples are concerned with the illustration of Theorem 8 and Theorem 9.

Example 1. Consider the following integral boundary value problem:

$$
\left\{\begin{array}{l}
{ }^{c} D_{0+}^{1.8} \varphi_{1.2}\left({ }^{c} D_{0+}^{1.5} x(t)\right)=1+\frac{1}{3} \sin t+\frac{e^{-t}}{\sqrt{10+t}} x^{0.2}(t), \quad t \in(0,1), \\
x(0)=\int_{0}^{1}\left(1+e^{-s}\right) x(s) d s, \quad x(1)=0, \\
\varphi_{1.2}\left({ }^{c} D_{0+}^{1.5} x(0)\right)=\varphi_{1.2}\left({ }^{c} D_{0+}^{1.5} x(1)\right)=\int_{0}^{1} \frac{1}{\sqrt{100+s^{2}}} \varphi_{1.2}\left({ }^{c} D_{0+}^{1.5} x(s)\right) d s .
\end{array}\right.
$$

The problem (70) can be rated as the boundary value problem where $\alpha=1.5, \beta=1.8, p=1.2$,

$f(t, x)=1+(1 / 3) \sin t+\left(e^{-t} / \sqrt{100+t}\right) x^{0.2}, a(t)=1+(1 / 3)$ $\sin t, b(t)=\left(e^{-t} / \sqrt{10+t}\right), g(t)=1+e^{-t}, \quad$ and $\quad h(t)=(1 /$ $\left.\sqrt{100+t^{2}}\right)$. By simple calculation, we have $\|a\|=4 / 3,\|b\|$ $=1 / \sqrt{10},\|g\|=2,\|h\|=1 / 10$, $\sigma_{1}=\int_{0}^{1}(1-s) g(s) d s \approx 0.87 \neq 1$, and $\sigma_{2}=\int_{0}^{1} h(s) d s \approx 0.1 \neq 1$. 
So, testing whether the hypothesis (H1) holds or not, we get

$$
\begin{aligned}
A & =\left[\frac{1}{\Gamma(\alpha)}\left(1+\frac{\|g\|}{\left|1-\sigma_{1}\right|}\right)\right]^{p-1} \frac{1}{\Gamma(\beta)}\left(1+\frac{\|h\|}{\left|1-\sigma_{2}\right|}\right) \\
& \approx 2.13, A\|b\| \approx 0.67<1 .
\end{aligned}
$$

Therefore, by Theorem 8 , the problem (70) has at least one solution.

Example 2. Consider the boundary value problem

$$
\left\{\begin{array}{l}
{ }^{c} D_{0+}^{1.7} \varphi_{1.5}\left({ }^{c} D_{0+}^{1.3} x(t)\right)=0.4 t+0.1 \sin (x(t)), \quad t \in(0,1) \\
x(0)=\int_{0}^{1} s x(s) d s, \quad x(1)=0 \\
\varphi_{1.5}\left({ }^{c} D_{0+}^{1.3} x(0)\right)=\varphi_{1.5}\left({ }^{c} D_{0+}^{1.3} x(1)\right)=\int_{0}^{1} \frac{1}{10+s^{2}} \varphi_{1.5}\left({ }^{c} D_{0+}^{1.3} x(s)\right) d s
\end{array}\right.
$$

Comparing with the problem (1), it can be easily seen that $\alpha=1.3, \beta=1.7, p=1.5, f(t, x)=0.4 t+0.1 \sin x, a(t)=$ $0.4 t, b(t)=0.1, g(t)=t$, and $h(t)=1 /\left(10+t^{2}\right)$.

Since $\|a\|=0.4,\|b\|=0.1,\|g\|=1,\|h\|=0.1, \sigma_{1}=\int_{0}^{1} s(1$ $-s) d s \approx 0.17 \neq 1$, and $\sigma_{2}=\int_{0}^{1}\left(1 / 10+s^{2}\right) d s \approx 0.1 \neq 1$, we obtain

$$
\begin{aligned}
|f(t, x)| & \leq 0.4 t+0.1|\sin x| \leq 0.4 t+0.1 \cdot \min \{1,|x|\} \leq 0.4 t+0.1 \cdot|x|^{0.5}, \\
A & =\left[\frac{1}{\Gamma(\alpha)}\left(1+\frac{\|g\|}{\left|1-\sigma_{1}\right|}\right)\right]^{p-1} \frac{1}{\Gamma(\beta)}\left(1+\frac{\|h\|}{\left|1-\sigma_{2}\right|}\right) \approx 1.91, \\
A\|b\| & \approx 0.19<1 .
\end{aligned}
$$

So, we can see that the hypothesis (H1) is satisfied. Calculating the radius $r$, we have

$$
r=\left(\frac{A\|a\|}{1-A\|b\|}\right)^{q-1} \approx 0.9
$$

Since $r<1$, for any $t \in[0,1]$ and any $x, y \in[-r, r]$, we get

$$
\begin{aligned}
|f(t, x)-f(t, y)| & =0.1|\sin x-\sin y| \leq 0.1|x-y| \\
M_{0} & =\left(1+\frac{\|h\|}{\left|1-\sigma_{2}\right|}\right)\left(\|a\|+\|b\| r^{p-1}\right) \approx 0.55 \\
\theta & =\frac{L(q-1) M_{0}^{q-2}}{\Gamma(\alpha) \Gamma(\beta)}\left(1+\frac{\|g\|}{\left|1-\sigma_{1}\right|}\right)\left(1+\frac{\|h\|}{\left|1-\sigma_{2}\right|}\right) \\
& \approx 0.33<1 .
\end{aligned}
$$

From the above discussion, we can see that the hypothesis (H2) holds. Therefore, it follows by Theorem 9 that the problem (72) has a unique solution.

\section{Data Availability}

No datasets are generated or analyzed during this study.

\section{Conflicts of Interest}

The authors declare that they have no conflict of interest.

\section{Authors' Contributions}

All authors carried out the proof and conceived of the study. All authors read and approved the final manuscript.

\section{References}

[1] A. A. Kilbas, H. M. Srivastava, and J. J. Trujillo, Theory and applications of fractional differential equations, vol. 204, Elsevier, 2006.

[2] L. S. Leibenson, "General problem of the movement of a compressible fluid in a porous medium," Izvestiya Akademii Nauk Kirgizskoĭ SSR, vol. 9, pp. 7-10, 1983.

[3] R. Metzler and J. Klafter, "Boundary value problems for fractional diffusion equations," Physica A. Statistical Mechanics and its Applications, vol. 278, no. 1-2, pp. 107-125, 2000.

[4] I. Podlubny, Fractional Differential Equations, Academic Press, San Diego, 1999.

[5] S. G. Samko, A. A. Kilbas, and O. I. Marichev, Fractional Integrals and Derivatives: Theory and Applications, Gordon and Breach Science, Yverdon, Switzerland, 1993.

[6] C. Bai, "Existence and uniqueness of solutions for fractional boundary value problems with p-Laplacian operator," Advances in Difference Equations, vol. 2018, no. 1, Article ID 862079, 2018.

[7] G. Chai, "Positive solutions for boundary value problem of fractional differential equation with p-Laplacian operator," Boundary Value Problems, vol. 2012, no. 1, 2012.

[8] T. Chen, W. Liu, and Z. Hu, "A boundary value problem for fractional differential equation with $p$-Laplacian operator at resonance," Nonlinear Analysis Theory,Methods \& Applications, vol. 75, no. 6, pp. 3210-3217, 2012.

[9] L. Hu and S. Zhang, "On existence results for nonlinear fractional differential equations involving the $p$-Laplacian at resonance," Mediterranean Journal of Mathematics, vol. 13, no. 3, article 544, pp. 955-966, 2016.

[10] X. Liu, M. Jia, and X. Xiang, "On the solvability of a fractional differential equation model involving the $p$-Laplacian operator," Computers \& Mathematics with Applications, vol. 64, no. 10, pp. 3267-3275, 2012.

[11] Y. Liu, D. Xie, D. Yang, and C. Bai, "Two generalized Lyapunov-type inequalities for a fractional $p$-Laplacian equation with fractional boundary conditions," Journal of Inequalities and Applications, vol. 2017, no. 1, 2017.

[12] H. Lu, Z. Han, S. Sun, and J. Liu, "Existence on positive solutions for boundary value problems of nonlinear fractional differential equations with $p$-Laplacian," Advances in Difference Equations, vol. 2013, no. 1, 2013.

[13] T. Shen, W. Liu, and X. Shen, "Existence and uniqueness of solutions for several BVPs of fractional differential equations with $p$-Laplacian operator," Mediterranean Journal of Mathematics, vol. 13, no. 6, article 766, pp. 4623-4637, 2016. 
[14] Y. Su, Y. Yun, D. Wang, and W. Hu, "Existence of solutions to nonlinear $p$-Laplacian fractional differential equations with higher-order derivative terms," Electronic Journal of Differential Equations, vol. 2018, no. 105, 2018.

[15] K. Jong, "Existence and uniqueness of positive solutions of a kind of multi-point boundary Value problems for nonlinear fractional differential equations with $p$-Laplacian operator," Mediterranean Journal of Mathematics, vol. 15, no. 3, article 1155, 2018.

[16] Y. Tian, S. Sun, and Z. Bai, "Positive Solutions of Fractional Differential Equations with -Laplacian," Journal of Function Spaces, vol. 2017, Article ID 3187492, 9 pages, 2017.

[17] J. Xu and D. O'Regan, "Positive Solutions for a Fractional pLaplacian Boundary Value Problem," Filomat, vol. 31, no. 6, pp. 1549-1558, 2017.

[18] S. Yao, G. Wang, Z. Li, and L. Yu, "Positive solutions for threepoint boundary value problem of fractional differential equation with $p$-Laplacian operator," Discrete Dynamics in Nature and Society, vol. 2013, Article ID 376938, 7 pages, 2013.

[19] Z. Han, H. Lu, and C. Zhang, "Positive solutions for eigenvalue problems of fractional differential equation with generalized $p$ Laplacian," Applied Mathematics and Computation, vol. 257, pp. 526-536, 2015.

[20] W. Jiang, "Solvability of fractional differential equations with p-Laplacian at resonance," Applied Mathematics and Computation, vol. 260, pp. 48-56, 2015.

[21] Z. Liu and L. Lu, "A class of BVPs for nonlinear fractional differential equations with $p$-Laplacian operator," Electronic Journal of Qualitative Theory of Differential Equations, vol. 2012, no. 70, pp. 1-16, 2012.

[22] X. Zhang and Y. Cui, "Positive solutions for fourth-order singular -Laplacian differential equations with integral boundary conditions," Boundary Value Problems, vol. 2010, no. 1, Article ID 862079, 2010.

[23] X. Zhang, M. Feng, and W. Ge, "Symmetric positive solutions for $p$-Laplacian fourth-order differential equations with integral boundary conditions," Journal of Computational and Applied Mathematics, vol. 222, no. 2, pp. 561-573, 2008.

[24] D. R. Smart, Fixed Point Theorems, Cambridge University Press, London, UK, 1980. 